\title{
TOWARDS VERSATILE SIMULATION MODELS OF TRANSPORT NODES
}

The paper deals with analysis of characteristics of simulation tools and models which are required for support of dispatcher's decisions in real operation, training of dispatchers and education. Further, a concept of simulation tool is discussed that will allow the construction and development of simulation models in which support for re/design of railway system, its management and also the training of dispatchers will be integrated.

Keywords: On-line simulation, operative control, operational planning

\section{Introduction}

Simulation methods currently represent widespread techniques supporting optimisations related to railway systems. The mentioned optimisations mainly pay essential attention to infrastructure design, to organisation of technological processes as well as to compositions and dimensions of various kinds of resources. Dominant utilisation of simulation tools supports tactical (middle-term) and strategic (long-term) planning related usually to infrastructural or operational proposals, which are supposed to guarantee optimal (or at least effective) behaviour of modelled system from the long-continuing (statistical) point of view. That traditional simulation support is included within the frame of so called off-line planning.

A simulation model (simulator) supporting off-line planning is based on "artificial" inputs and not on interconnections with a real-time operation. Hence an important role, within the stage of simulation model building, is played by a collection and analysis of input data. The most common procedure is to realise a statistical analysis of historical or prognostic data and on this base to implement corresponding input generators (this approach is called input analysis). Another (less commonly used) way how to realise simulator's inputs is to utilise an identical input flow which occurred in the past in reality (this approach is denoted as data-driven or trace-driven simulation).

The most important part, and from the implementation viewpoint also the most difficult, is represented by processes' control modelling. The control in this sense can be understood as a sequence of decisions which react to occurrence of identified problems (a problem is here interpreted as intervention of a control element) In the case that a modelled system is purely deterministic (does not contain any randomness) it is quite easy to realise control following a fixed plan. This plan is composed of anticipated reactions (decisions) to an expected sequence of occurrences of specified problems in concrete time instants (within the frame of a planning period).
The mentioned kind of plan can be defined for instance in the form of a flowchart (e.g. using formalism of Petri nets as models of simulator's decision-making mechanisms [7]. An example is depicted on Fig. 1.

Substantially more difficult case is related to a situation when the problem occurrence is of a stochastic character, i.e. the time instant of a problem's appearance is not predictable and so it is not possible to plan a corresponding reaction in advance. A control element takes a decision when a given problem was identified either just in a moment of its occurrence or slightly in advance such a character of decision-making process is called an operative control. Apparently a solution of such problem is not possible to fold directly in any plan.

Modelling of operative control compared with modelling of control by a fixed plan represents a considerably more complex task. In the field of modelling and simulation, a significant attention is paid to research and development of appropriate techniques focused on modelling of operative control, i.e. modelling of an intelligent decision-making related to managing workers.

\section{Short-term planning in a factory railway system}

The character of railway transportation systems, from the mentioned point of view, seems to be mixed. In some kinds of railway systems dominates an operation using a fixed plan (e.g. modern marshalling yards [1] or passenger stations) and in others an operative control plays a key role (e.g. factory railways or port sidings).

Let us take a factory railway as an illustrative example. The operation of factory railway has to cope with the occurrence of many input flows' irregularities, which is not the case in other kinds of railway nodes. It means that the operative control is quite typical for a factory railway operation. On the other hand there exists no agreement between the railway company (usually operating on a relatively large geographic territory) and connected factory railway,

\footnotetext{
* Norbert Adamko ${ }^{1}$, Antonín Kavička ${ }^{2}$, Valent Klima ${ }^{1}$

${ }^{1}$ University of Žilina, Faculty of Management Science and Informatics, Tel. +421 415134222 , E-mail: Norbert.Adamko@fri.utc.sk,

Valent.Klima@fri.utc.sk

${ }^{2}$ University of Pardubice, Jan Perner Transport Faculty , Tel.: +420 466036 645, E-mail: Antonin.Kavicka@upce.cz
} 


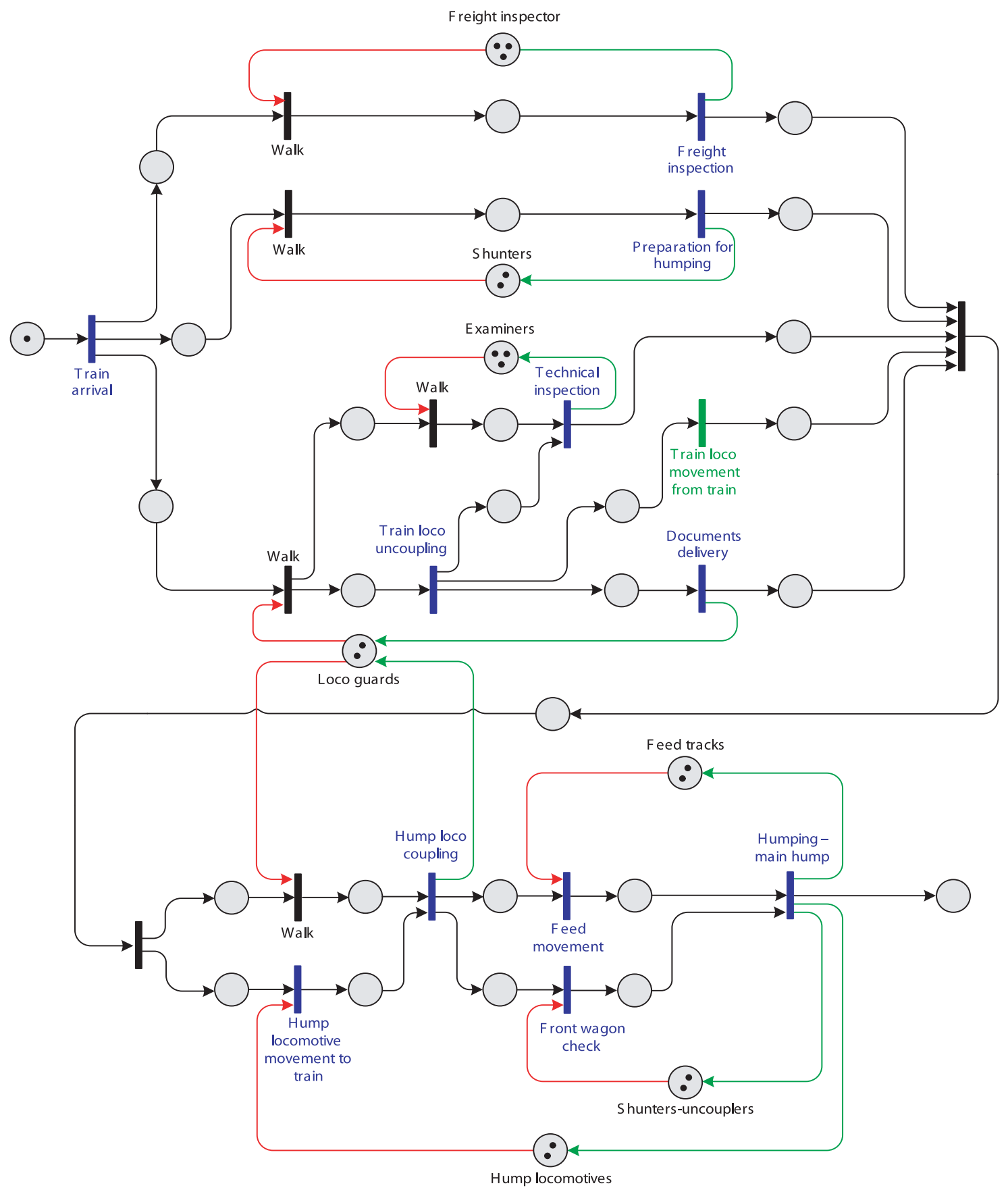

Fig. 1 Petri net as a model of service plan related to trains arrived in marshalling yard

which would be based on the sophisticated plan reflecting effective utilisation of resources belonging to both subjects. So, there is an urgent question: "How to prepare (with the help of simulation) an appropriate operational plan respecting requirements of both concerned parties?" The simulation model (with the motivation to consider a quality of strategic and tactical intensions) has to include a lot of operative decisions and, therefore, a resulting plan is made on a lower level of robustness, i.e. its application for the factory railway within another time interval (another day, week etc.) is less likely. In addition the plan construction represents a highly time-consuming process. Operative decisions are in this case understood as simulation model features reflecting essential reactions to the input data. For example, an experimenter has to decide where to displace wagons (because of capacity problems), which can't be shunted to unloading places. This situation can occur for example once a month. There are a lot of problematic situations within factory railways, which are only rarely similar. 
Let us mention several factors negatively affecting a robustness of the factory railway operational plan:

- Irregular composition of incoming flows of wagons which may require additional shunting and, therefore, prompts the use of additional resources.

- Acyclic working regime of manipulation resources related to loading-unloading places.

- Shunting in connection with a preparation of a wagon set before attendance and a consequent loading-unloading on the specialised places -a configuration of trackage (spur tracks in factory halls) as well as an order of individual wagons within the incoming trains are essential.

- Conflict between rail and road freight traffic due to requirements for manipulation resources and for the same loading-unloading places.

- Reaction to operative client's demands related either to specific kind of wagon or consignment.

- Conflicts on the railway crossings.

A modification of input conditions (data) in order to reach a higher level of operational plan robustness already represents the way of problem solutions (e.g. eligible timetable of incoming trains, adequately pre-sorted incoming trains etc.). It is important to realise what properties are expected from a simulation tool to be effectively applicable to the construction of a factory railway operational plan, i.e. which of its features can compensate longer preparation of less flexible plans. The simulation tool has to enable very quick plan modifications and subsequently their verification for the purpose of strategic and tactical planning. The following tool features support the mentioned requirements:

- Transparent entering of succession of individual steps related to preparation of wagon set before the corresponding attendance and operation on loading-unloading places. It is essential to support all infrastructural input data by graphical editor displaying the trackage. In addition, simulation tool editors should dispose of automatic routines focused on filling out required parameters according to initial partial data defined by the user.

- Possibility to create macros which represent frequently repeating standard procedures, e.g. transposition of empty wagons to an available track in factory hall and their loading. In the case it is needed to repeat the mentioned procedure, a corresponding macro is called and it is not necessary to pay attention to individual steps any more. Actual experience indicates that a definition of macros' palette should be prepared for each factory railway model individually because each factory railway disposes of unique trackage configuration.

It is quite complicated to compare the use of a simulation for the optimisation of a factory railway operational plans to other kinds of railway nodes, which usually process higher quantity of wagons (marshalling yards, passenger stations etc.). In spite of this fact, we can offer the examples of tasks which can be successfully solved with the help of simulation and bring evident benefits for the factory railways as well as for the surrounding railway network:

- Modelling of consequences related to a growing production (input and output flows rate changes).
- Investigation of changes associated with train timetables and operational plans and their potential optimisation.

- Rationalisation of the fleet of mobile resources - shunting locomotives, personnel, manipulation resources.

- Considerations of intended investments in the infrastructure or in the mobile resources versus operational costs.

\section{On-line simulation and information system}

Let us ask a question if once constructed, verified and validated, simulation model can be helpful as a control support (with required modifications) even after implementation of a real system. This situation can be understood as "a disconnection" of the simulator from artificial, statistically obtained data, and "a connection" to real data which are continually produced by a real system. Running such a simulation model represents so called on-line simulation, which can support operational planning and control.

The concept of the on-line simulation differs from the classical off-line simulation mainly due to the following features:

- Simulation model is directly connected with a real system (e.g. with information/disposition real-time operating software system) and the values of its selected (input) state variables are updated directly from reality (it is a special case of data-driven simulation).

- Duration of individual simulation trials should be substantially faster than realisations of corresponding processes in reality. Such condition is required because the proposed solutions (plans) based on the results of the simulation trials have to be still feasible within the current real situation.

So, the workers from operative control level expect from the on-line simulation some kind of a support serving for analysis of actual status of a real system and consequently for the construction of short-term operational plans (on-line planning), which are subsequently applied within the real system (e.g. scheduling and rostering of resources etc.).

What methodology is supposed to be applied for the needs of the on-line planning? There are principally two approaches suitable for that purpose. The first one is based on the rule that new planning is made only in the case when the current plan substantially differs from reality and hence it is not applicable any more (this concept is called reactive planning). The second approach called proactive planning makes trials with the aim to improve an existing plan. The simulation run is executed periodically to look for potential problems which can occur in the near future. The basis for plan's construction or modification is formed by results of series of simulation trials which are focused on the different prospective operational scenarios originating from the present state of a real system.

In the on-line simulation there is not enough time for evaluation of the prospective scenarios. Hence the experimenter has to use a strategy, which enables to use relevant findings from the simulation trial, which are still valid in the operational situation. 
It was mentioned above that an on-line simulator is directly connected to a real process. However, the typical implementation of such a connection is realised via a real-time information system (updated directly from real processes), which represents a source of data for the on-line simulator. From that point of view, an online simulation model can be understood as an integration of a simulation model with a corresponding information system [2]. Then it is obvious that the success of an on-line simulation highly depends on the quality of the relevant information system. In the case of a factory railway, the real-time information system has to contain data about

- accurate position of each piece of the rolling stock,

- incoming and outgoing trains and their compositions,

- routings of wagons within the frame of each sorting stage etc.

It can be said that properties of all advanced contemporary railway information systems are quite close to the requirements of an on-line simulation.

Thanks to integration of the on-line simulator and information system it is possible to evaluate prospective operation scenarios and in addition to it, produce even reconstruction of processes realised in the past. This is so called retrospective simulation and it can be very useful for the analysis of critical events caused either by a control element or technical-technological deficiency or unexpected character of incoming flows of wagons/trains.

\section{Using simulation model in the process of training and education}

Another alternative utilisation of simulation tools and simulation models is in the area of vocational training courses for dispatchers and educational courses at schools. It is evident that the dispatcher shares the responsibility related to the railway node operation and so he has to be properly trained before starting his vocation. This process is costly and quite time consuming. It is impossible to tackle theoretically all problems which can be encountered in practice. It means that the dispatcher starting his job will probably meet situations which were not dealt with in his training and therefore there is quite a big potential of wrong decisions with negative consequences (not only from an economic viewpoint). Therefore it would be useful to encourage dispatchers to study their nodes not only under standard conditions but also in exceptional situations. Certainly it is not admissible to make experiments within a real operation because of economical and safety reasons (e.g. it is not permissible to cause a crisis merely for the training of dispatchers).

Simulation models can represent very convenient tools and environments, within the frame of which the dispatchers can investigate a railway node under various situations including critical ones. Moreover, important infrastructural and operational changes and their consequences can also be studied. Let us mention main advantages of utilisation of simulation tools within the dispatchers' training:
- Detailed introduction to a concrete node and its operation under various conditions (infrastructure, incoming flows, technological processes, performance parameters, node limits etc.).

- Possibility to train an operative control of a railway node even under crisis conditions.

- Experimentation with different variants of technological processes applied within a railway node.

- Prompt verification of the consequences related to the just taken decisions.

- Subsequent analysis of quality of the dispatcher's decisions made by a supervisor.

- Relatively low training costs.

The simulation model reflects a real system (railway node) and its operation within the computer environment. In addition, the simulator intermediates a depiction of permanent and temporary elements and focuses also on modelling of decision-making processes. It means in fact that the simulator performs activities which correspond to the dispatcher's real actions. Utilisation of a simulation model as a training tool enables substitution of a real dispatcher by a trained dispatcher. The simulator presents to a trained person all relevant data needed for a decision-making process (run-time animation outputs, statistical observations, graphs etc.) and on the other hand it is able to accept those decisions. Such a simulator is called a interactive simulation model.

The simulation model is able to identify a set of potential problems which are supposed to be solved during a simulation trial. The user (a trained dispatcher) selects from the palette those of identifiable problems whose solution he would like to carry out.

When such a problem occurs the simulator offers potential alternative solutions (if they exist) and the user can choose one of them according to his proper considerations. That decision is then accepted and it represents an integral part of simulation trial. The problems not solved by a user are solved by integrated software algorithms. The mentioned flexible approach leads the user to focus only on the problems which he is interested in and not on currently unsubstantial ones.

It should be emphasised that even the most accurate and detailed simulation model cannot be identical with reality and entirely substitute real operational experience. However, there is no doubt that the mentioned kinds of simulators can represent a suitable part in a complex educational programme for railway node dispatchers.

\section{Conclusion}

The prospective computer simulation, focused on a railway system design and its operation, will obviously encompass simulation models integrating individual supporting stages (design planning - control - training) depending on the ongoing quality of corresponding information systems. In order to successfully manage the mentioned integrated approach it is essential to utilise an appropriate flexible architecture of a simulation model. The authors 
have been cooperating on the development of such a simulation support with the help of original agent-based simulator's architecture [8].

\section{Acknowledgment}

This work has been supported by the Slovak grant foundation under grant No. 1/1049/04 "Agent Oriented Architecture of Service
Systems Simulation Models" and by the National Research Program of Czech Republic under project No. MSM 0021627505 "Theory of Transportation Systems".

\section{References}

[1] KLIMA, V., KAVIČKA, A.: Simulation support for railway infrastructure design and planning processes, In Proceedings of COMPRAIL 2000 conference in Bologna - Italy, WIT-Computational Mechanics Publications, Southampton-UK, September 2000, pp.447-456

[2] BANKS, J. edit.: Handbook of Simulation, John Wiley\&Sons, New York, 1998

[3] ORTÚZAR, J. D., WILLUMSEN, L., G.: Modelling Transport. 3. ed. John Wiley\&Sons, 2004

[4] ADAMKO, N., ZAŤKO, M., KLIMA, V., KAVIČKA, A.: Alternative possible utilisation of simulation tools focused on railway operation, In Proceeding of international symposium “ŽEL 2002, Žilina, pp. 154-159, (in Slovak)

[5] HENSHER, D., A., BUTTON, K., J. edit.: Handbook of Transport Modelling. Pergamon, 2000.

[6] XU, Z. L., KLIMA, V., KAVIČKA, A.: The Realization of Joint Terminal Process Simulation by Computer, Railway Transport and Economy, China Academy of Railway Sciences, Beijing, 1/2003, pp. 51-53 (in Chinese)

[7] KAVIČKA, A.: Petri net with decision transitions applied within ABAsim architecture of simulation model (in Czech), In Proceedings of the 37th international conference "MOSIS '03”, editor-MARQ Ostrava, 2003, pp. 373-380

[8] ADAMKO, N., KLIMA, V., KAVIČKA, A., LEKÝR, M.: Flexible hierarchical architecture of simulation models, In Proceedings of European simulation and modelling conference, Eurosis, Paris, 2004, pp. 30-34. 